\title{
Mechanism of the Gas-Liquid Two-Phase Chaotic Flow in Single Fracture
}

\author{
Dong Yang, ${ }^{1,2}$ Zhiqin Kang $\mathbb{D}^{1,2}$ and Yangsheng Zhao ${ }^{1,2}$ \\ ${ }^{1}$ Key Lab of In-Situ Property-improving Mining of Ministry of Education, Taiyuan University of Technology, Taiyuan, China 030024 \\ ${ }^{2}$ The In-Situ Steam Injection Branch of State Center for Research and Development of Oil Shale Exploitation, Taiyuan 030024, China
}

Correspondence should be addressed to Zhiqin Kang; kangzhiqin810101@126.com

Received 8 June 2020; Revised 29 July 2020; Accepted 7 August 2020; Published 25 August 2020

Academic Editor: Yanlin Zhao

Copyright (c) 2020 Dong Yang et al. This is an open access article distributed under the Creative Commons Attribution License, which permits unrestricted use, distribution, and reproduction in any medium, provided the original work is properly cited.

\begin{abstract}
The seepage of gas-liquid two-phase flow in fracture is a commonly found phenomenon in nature. To reveal the underlying mechanism and the critical condition of the chaos occurrence, a stochastic gas-liquid two-phase flow seepage model is established, and then investigated through a numerical simulation and a horizontal Hele-Shaw experiment. The numerical simulation and laboratory experiment results show that the seepage chaos of gas-liquid two-phase flow takes place when the relative saturation is in the range of gas relative saturation $44 \%-70 \%$, and the occurrence probability can be expressed in polynomials. The chaos probability exceeds $80 \%$ when the relative saturation of gas is $47 \%-65 \%$, and the chaos probability is $100 \%$ when the relative gas saturation is $57 \%-60 \%$. It is found that the stochastic variation of gas connection cluster and the compressibility of gas lead to a remarkable change of pressure gradient of the gas-liquid flow both in magnitude and direction. Therefore, the turbulent flow is formed, the kinetic energy of fluid transport decreases gradually, and the flow is stopped at last.
\end{abstract}

\section{Introduction}

Gas-liquid two-phase fluids transport in a fracture is commonly seen in a variety of engineering applications, including coal bed methane extraction, hydrology, oil/gas resource exploitation, and geothermal utilization. The pioneering research along this line stemmed from the work of Tangren et al. [1] and Saffman and Taylor [2]. Corey [3] researched the interrelation between gas and oil relative permeability. Ishii [4] and Pai [5] published the books about two-phase flow. Then Hele-Shaw cell method has been extensively used to study the different status of gas-liquid two-phase flow in a fracture [6-11]. Thereafter, the gas-liquid two-phase flow in $\mathrm{mini} /$ micro gaps, narrow horizontal annuli, microchannels, and rock joint/fractures are continuously researched [1225] (Adolfo 2001, Donata 2008, Reid 2002). Some work revealed the complexity of gas-liquid two-phase flow. González et al. [26] researched the flow equations on a fractal structure. Faybishenko [27] studied the chaotic dynamics in flow through unsaturated fractured media. Schlueter et al. [28] researched the fractal dimension of pores in sedimentary rocks and its influence on permeability. Maria (2007) and Zhao (2007) adopted numerical methods to simulate the complex patterns of gas-liquid two-phase flow. Zhao et al. [29] has researched the transient pulse test and morphological analysis of single rock fractures, and later Zhao et al. [30, 31] also set up a numerical creep model to simulate the rheological fracture behavior of rock cracks subjected to hydraulic pressure and far-field stresses. By using experimental methods, Zhao et al. [32, 33] investigates fracture toughness and subcritical crack growth of three rocks shear behavior of infilled rock joints. These works have played a positive role in understanding the various mechanical behaviors of rock fractures, but are limited to the description of the solid mechanical behavior, and have not described the fluid characteristics of the two-phase fluid in the fractures. Although there are many other pieces of literature mentioned about two-phase flow in single fractures, but few concerned about the chaotic phenomenon of two-phase flow in single fractures.

From 1997 to 2000, Zhao et al. in Taiyuan University of Technology conducted a series of research in the seepage of 
gas-liquid two-phase flow in single rock fracture. They found that when the relative gas saturation felled into a given range, the two-phase fluid will not transport along with the definite direction, but turn out to be a nonregular and chaotic flow. The flow kinetic energy seems being consumed up and the total flow will stop.

This stationary condition cannot be changed even though the inlet pressure is increased. This special natural phenomenon may be called the chaos of gas-liquid two-phase flow in a fracture. The critical condition for its occurrence and the underlying mechanism are of most importance for understanding the problem and solve them in practice.

\section{Stochastic Mixing Seepage Model for the Transport of Gas-Liquid Two-Phase Flow in a Fracture}

For single-phase gas/liquid flow or the gas-liquid two-phase flow with high relative saturation of gas or liquid, the flow pattern in a fracture is a simple laminar flow. In the twophase seepage transport, the position of a given gas or liquid cluster at a given moment is stochastic and is hardly predicted by the existing theories. The governing equations of gas-liquid two-phase transport can be constructed via stochastic distribution of gas or liquid cluster with the following assumptions:

(1) The gas-liquid flow in a fracture is saturated flow, and their relative saturation of gas and liquid are

$$
S_{\mathrm{g}}=\frac{Q_{\mathrm{g}}}{\left(Q_{\mathrm{g}}+Q_{\mathrm{w}}\right)} S_{\mathrm{w}}=Q_{\mathrm{w}} /\left(Q_{\mathrm{g}}+Q_{\mathrm{w}}\right)
$$

(2) The interfacial tension between gas and liquid is neglected. In the whole fracture, the gas pressure and liquid pressure are equal at each point

(3) The two-phase flow is an isothermal process

(4) Any given position in the fracture is only occupied by single-phase gas or single-phase liquid

(5) The intersection of any flow in a fracture and the relative saturation of gas/liquid depends on the intersection pressure and the relative saturation of gas or liquid at the inlet

(6) The gas-liquid two-phase flow in a fracture can be idealized as a seepage process

Based on the above assumptions, the seepage transport equation of gas-liquid two-phase flow in a fracture can be obtained by coupling the mass conservation equation, energy conservation equation, and the state equation of gas.

Mass conservation equation of gas-liquid two-phase flow:

$$
\operatorname{div}\left(\rho_{\mathrm{w}} \vec{q}_{\mathrm{w}}+\rho_{\mathrm{g}} \vec{q}_{\mathrm{g}}\right)+\frac{\partial\left(\rho_{\mathrm{w}} S_{\mathrm{w}}+\rho_{\mathrm{g}} S_{\mathrm{g}}\right)}{\partial t}+I=0
$$

The constitutive equation of gas seepage in a fracture:

$$
q_{\mathrm{g}}=-\frac{b^{2}}{12 \mu_{g}} S_{g} \frac{\partial p}{\partial s}
$$

The constitutive equation of liquid seepage in a fracture:

$$
q_{\mathrm{w}}=-\frac{b^{2}}{12 \mu_{\mathrm{w}}} S_{\mathrm{w}} \frac{\partial p}{\partial s}
$$

The relative saturations of gas and liquid in two-phase flow are

$$
\begin{gathered}
S_{\mathrm{g}}=\frac{p_{0} S_{\mathrm{g} 0}}{p\left(1-S_{\mathrm{g} 0}\right)+p_{0} S_{\mathrm{g} 0}}, \\
S_{\mathrm{w}}=1-S_{\mathrm{g}} .
\end{gathered}
$$

The equation of state of ideal gas is

$$
\rho_{\mathrm{g}}=\frac{p M}{R T}
$$

Upon substitution of equations (2), (3), (4) and (5) into equation (1) and after rearrangement, the governing equation of gas-liquid two-phase flow in fracture could be written as

$$
\begin{gathered}
\left(\frac{\rho_{\mathrm{w}} b^{2}}{24 \mu_{\mathrm{w}}} \frac{\left(1-S_{\mathrm{g} 0}\right)}{p\left(1-S_{\mathrm{g} 0}\right)+p_{0} S_{\mathrm{g} 0}}+\frac{M}{R T} \frac{b^{2}}{24 \mu_{\mathrm{g}}} \frac{p_{0} S_{\mathrm{g} 0}}{p\left(1-S_{\mathrm{g} 0}\right)+p_{0} S_{\mathrm{g} 0}}\right)\left(\frac{\partial^{2} p^{2}}{\partial x^{2}}+\frac{\partial^{2} p^{2}}{\partial y^{2}}\right) \\
\left(\frac{M}{R T} p-\rho_{\mathrm{w}}\right) \frac{\partial}{\partial t}\left(\frac{p_{0} S_{\mathrm{g} 0}}{p\left(1-S_{\mathrm{g} 0}\right)+p_{0} S_{\mathrm{g} 0}}\right)+\frac{M}{R T} \frac{p_{0} S_{\mathrm{g} 0}}{p\left(1-S_{\mathrm{g} 0}\right)+p_{0} S_{\mathrm{g} 0}} \frac{\partial p}{\partial t},
\end{gathered}
$$

where $\rho_{\mathrm{g}}, \rho_{\mathrm{w}}$ are gas density and liquid density, respectively; $S_{\mathrm{g}}, S_{\mathrm{w}}$ are the relative saturation of gas and liquid; $R$ is universal gas constant; $T$ is absolute temperature; $p$ is fluid pressure; $\mu_{\mathrm{g}}, \mu_{\mathrm{w}}$ are dynamic viscosity of gas and liquid; $S_{\mathrm{g} 0}$ is the relative gas saturation when the fluid pressure is $p_{0}$. Equation (7) is a complex nonlinear Partial Differential Equation in that both the numerator and denominator of its coefficient contain the unknown variable $p$.

\section{Numerical Simulation of Gas-Liquid Two- Phase Flow in a Fracture}

3.1. Solving Tactic and Simulation Procedure. The Finite Element Method (FEM) is employed to solve equation (7). The procedures are as follows: (1) Divide the flow field in a fracture into quadrilateral grid; (2) Iterate over time to solve the discrete equation. Because there exists a variable $p$ in the coefficient, the equation at the time $t_{0}+d t$ is solved with the value of $p$ at the time $t_{0}$ by iteration method. When the critical tolerance is reached, we proceed to the next time step; (3) in the whole cycle, the relative gas saturation of each intersection of flow is calculated at the end of each iteration step. 
(i) Numerical simulation model: the model is $500 \mathrm{~mm}$ long and $100 \mathrm{~mm}$ wide, as shown in Figure 1. The four sides' boundary condition is stationary, that is, $q=0$ at where $x=0$ and $x=L$. The pressure at the inlet is given as $p_{1}$, the pressure $p_{2}$ at the outlet equals $1 \mathrm{~atm}$

The value of the parameters in the calculation:

(1) The dynamic viscosity of water and gas are $\mu_{\mathrm{w}}=$ $0.001009 \mathrm{~Pa} \bullet \mathrm{s}, \mu_{\mathrm{g}}=0.000018 \mathrm{~Pa} \bullet \mathrm{s}$, respectively

(2) The absolute temperature: $T=273 \mathrm{~K}$

(3) Gravitational acceleration: $g=980 \mathrm{~cm} / \mathrm{s}$

(4) Water density: $\rho_{\mathrm{w}}=1 \mathrm{~g} / \mathrm{cm}^{3}$

(5) Universal gas constant: $R=8.3144598, \mathrm{~J} /(\mathrm{mol} \cdot \mathrm{K})$

(ii) Numerical simulation procedure: The area is divided into 50000 elements. The fracture widths are $0.1 \mathrm{~mm}$ and $0.2 \mathrm{~mm}$, and the inlet pressures are $1 \mathrm{MPa}$ and $2 \mathrm{MPa}$, respectively. We performed a whole calculation at each gas saturation. In a rough calculation, the gas saturation is given at an interval of $2 \%$, but in the gas saturation range for extraordinary flow the interval of relative gas saturation is $1 \%$

(iii) The chaos criterion of gas-liquid flow in a fracture: The gas-liquid two-phase flow in a fracture generally performs a seepage process. However, it sometimes performs chaotic flow, in which the flow pattern in the fracture is disorder and stops suddenly. Mathematically, this shows that the solution of the equation diverges or the solver fails. The criterion of this simulation is that the chaos occurs when the square power of pressure gets to be negative, that is, $p^{2}<0$

3.2. The Criterion Parameters of the Chaos in the Gas-Liquid Two-Phase Flow in a Fracture. To reveal the mechanism of the chaos of the gas-liquid two-phase flow, the simulation was carried out in an interval of $1 \%$ in the saturation ranging from $40 \%$ to $80 \%$. Because of the stochastic distribution of the position of the gas and liquid, the chaos probability for a given gas saturation is calculated after iterating for 100 times under this saturation. By simulation, it is found that the chaos of the gas-liquid flow mostly occurs in the gas saturation of $44 \%$ $70 \%$. In the gas saturation ranging from $56 \%$ to $62 \%$, the probability of the chaos is $80 \%$. In the gas saturation ranging from $57 \%$ to $60 \%$, the probability of the chaos is $100 \%$. The simulation indicates that the chaos probability changes with gas saturation in forth power polynomial, as shown in Figure 2.

$p\left(S_{\mathrm{gc}}\right)=-0.0007 S_{\mathrm{g}}^{4}+0.1461 S_{\mathrm{g}}^{3}-12.142 S_{\mathrm{g}}^{2}+460.05 S_{\mathrm{g}}-6609.7$

Its fitted correlative coefficient is about $97.8 \%$; the maximum value of this model is multisolution. This shows that the criterion parameter is in the range.

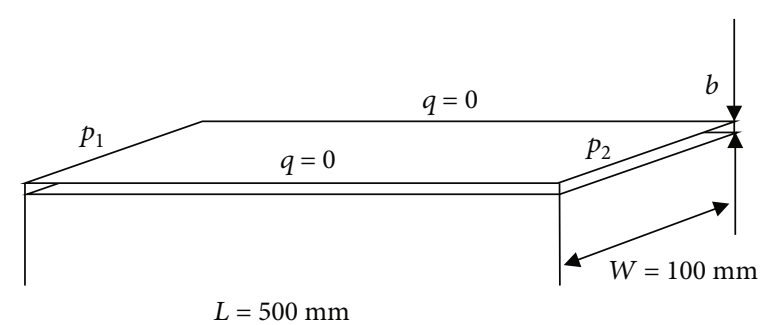

FIGURE 1: Sketch map of numerical simulation model.

3.3. The Mechanism of the Chaos of Gas-Liquid Two-Phase Flow in Fracture. In the gas-liquid flow in fracture, the gas or liquid distributes randomly, the cluster has variable sizes, and shapes form continuously. With the gas saturation being $32 \%-50 \%$, the size of the water cluster changes vastly, but the size of the gas cluster changes little and its distribution remains stable. In the gas saturation of $50 \%-70 \%$, the size of the water cluster changes marginally, but the size and shape of the gas cluster change significantly. Due to the gas compressibility and big change of the gas cluster, the pressure magnitude and direction changes vastly in the two-phase flow process. This explains the chaos phenomenon. Figure 3 shows the pressure gradient distribution in a fracture for the gas saturation of $30 \%$. It can be seen that in both the whole figure and local figure the flow direction is uniform, and the flow state is ordered, definite and steady.

Figure 4 shows the ten largest connected clusters of gas. Gas is dispersed randomly in water in the fracture. Part of gas has formed a complex connected cluster in water.

Figure 5 shows the pressure gradient vectors in fracture for the relative gas saturation of $60 \%$. It can be seen in both the whole figure and local figure that the flow in this area is disorder and in mass, that is, so-called chaos phenomenon. This flow pattern decreases the energy and then stops quickly. This is the chaos mechanism of the gas-liquid twophase flow in fracture.

Analysis of the pressure distribution of flow in fracture: In Figure 6(a), the fluid pressure changes slightly along the flow path when the relative gas saturation is $30 \%$. In Figure 6(b), the pore pressure declines steeply in several sections and the pressure gradient approaches to zero in some sections when the relative gas saturation is $60 \%$. This indicates that in this case, the chaos phenomenon of two-phase fluid transfer starts to appear.

3.3.1. Analysis of the Size of Max-Cluster Changing with the Relative Gas Saturation. For different gas saturation, the $500 \times 100$ grid elements are given to represent gas or liquid quality randomly, and then the ratio between the grid number occupied by gas max-cluster and the grid number occupied by liquid max-cluster is calculated by percolation theory, as shown in Figure 7. In this figure, the liquid cluster occupies most seepage space for the gas relative saturation less than $33 \%$ and gas cluster for the gas relative saturation great than $70 \%$. When the gas relative saturation is between 50 and $70 \%$, the size of the biggest gas cluster changes vastly, the flow direction changes vastly 


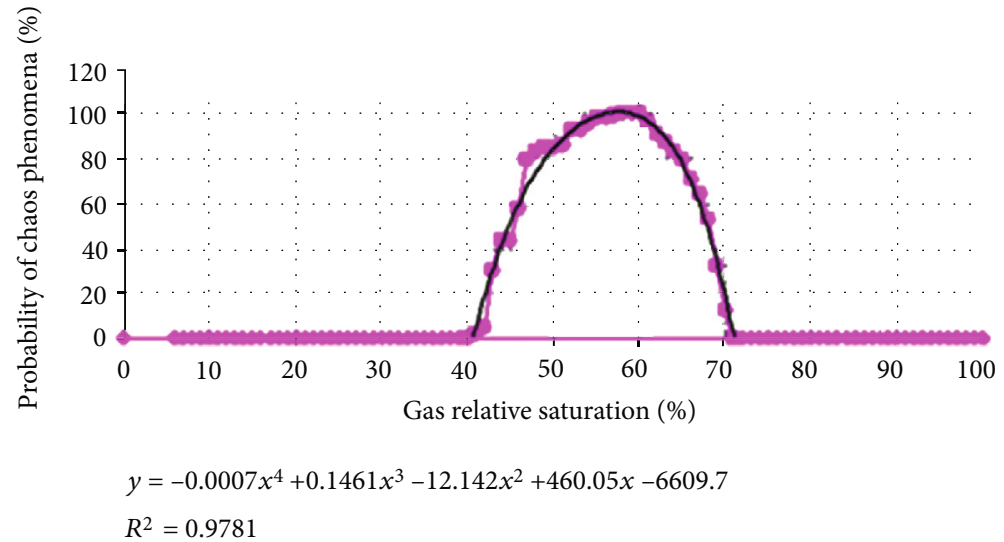

FIGURE 2: The probability of chaos phenomena happening of transport vs. Ga relative saturation.

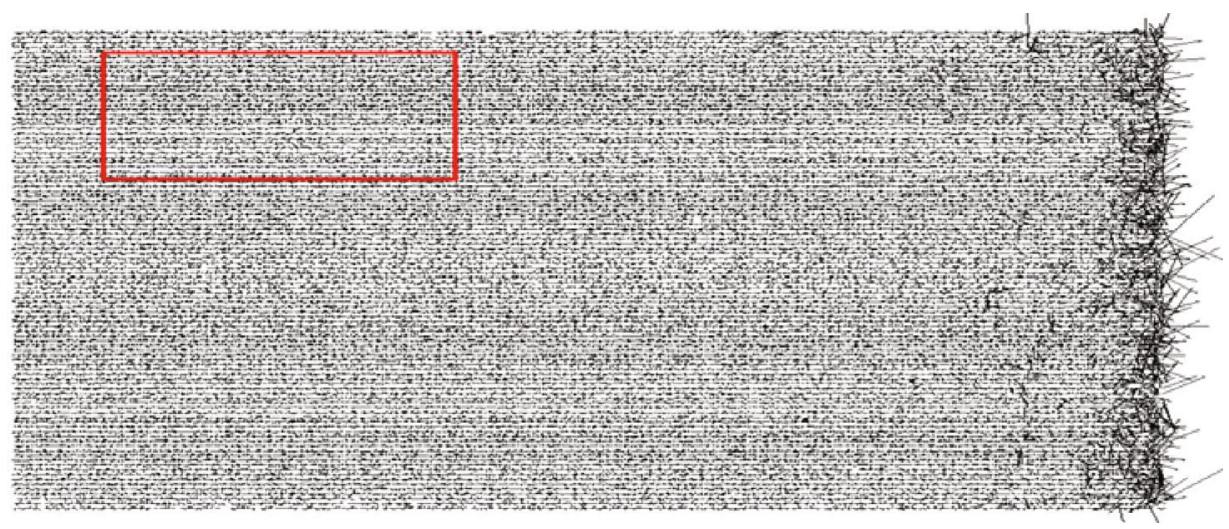

FiguRE 3: The pressure gradient distribution of the flow in the fracture at the gas saturation of $30 \%$.

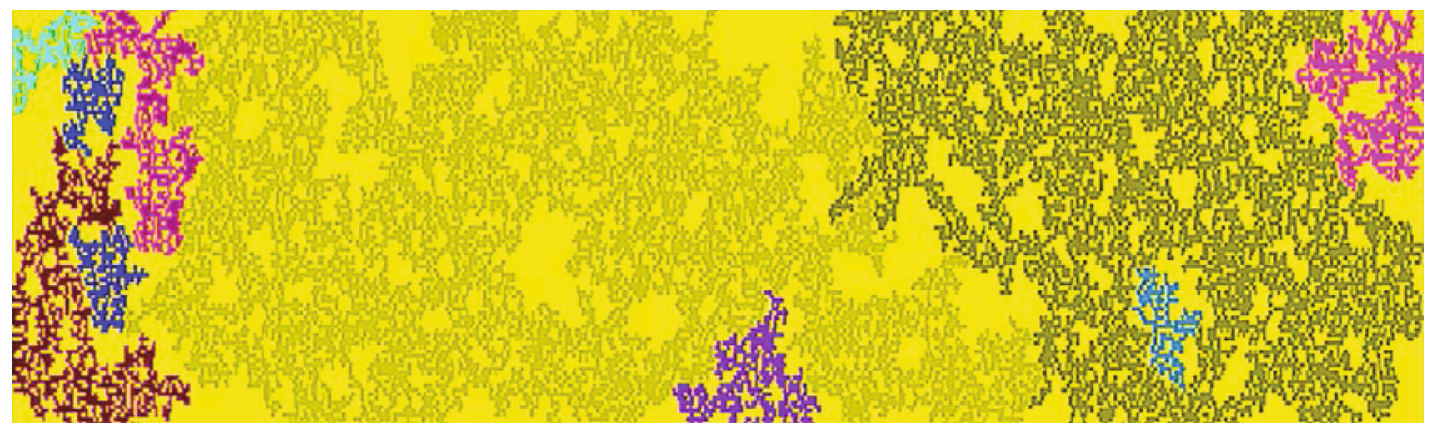

FIgURE 4: The gas connection clusters (including only the ten largest clusters which show in different color).

due to the gas compressibility, and the flow becomes turbulent. The flow energy is consumed up and the chaotic flow occurs.

Because of the great difference of seepage properties of gas and liquid, and influenced by the gas compressibility and repeated compress and expansion of the gas microcluster under the pressure, the energy of the gas is restored and released repeatedly. This makes the flow in fracture unstable and the micro-clusters mixed with gas and liquid are formed. When the gas relative saturation gets to the critical criterion, the gas-liquid two-phase flow becomes chaos, the flow direction is nonuniform, the flow energy is consumed up, and the flow stops. This is the mechanism of the chaos of gas-liquid two-phase flow in fracture.

\section{The Horizontal Hele-Shaw Experiment of the Chaos of Gas-Liquid Flow in a Fracture}

4.1. Experimental Apparatus. The modified horizontal HeleShaw experiment setup is employed in this research. The configuration of the setup is given in Figure 8, and Figure 9 is its photograph. 


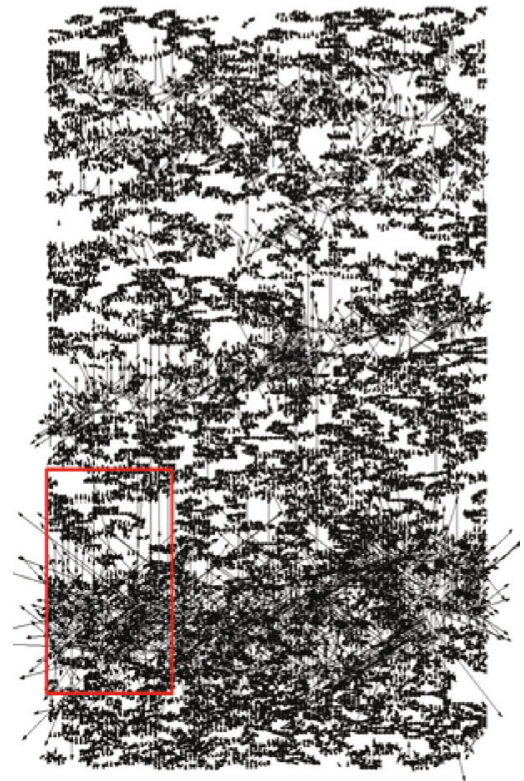

(a) The pressure gradient

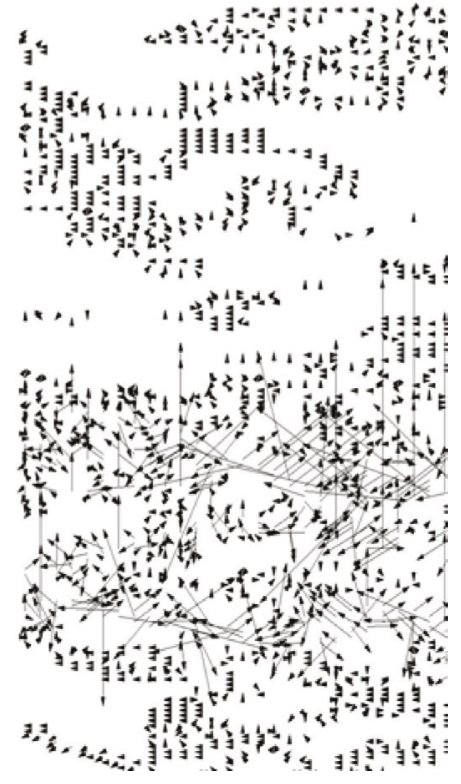

(b) Partial enlarged detail of (a)

Figure 5: The pressure gradient of the chaotic flow at the gas saturation of $60 \%$.

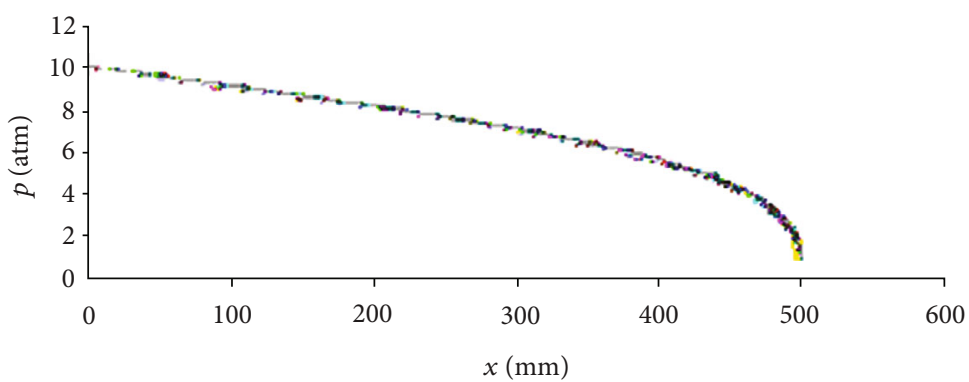

(a) The relative gas saturation is $30 \%$

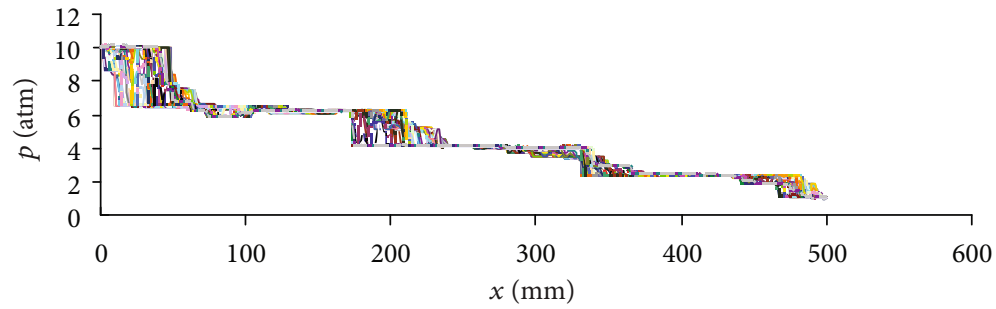

(b) The relative gas saturation is $60 \%$

FIGURE 6: The pressure distribution in fracture.

To ensure the consistent water supply and the stability of the gas and liquid pressure, the water was injected by gas pressure. The gas and water were mixed and injected into the fracture after passing the one-way valves, respectively. Several filters were set in the water pipe and gas pipe to prevent the dirt from going into the fracture and affecting the testing result. The gas emission was measured by collecting gas by the draining water method. When the gas emission and water discharge were measured, the pressure at the inlet and inside the fracture was tested, and the typical flow patterns were photographed by a high-resolution camera for further detailed analyzing.
The modified experiment setup consists of a Hele-Shaw simulation system, a gas-liquid mixed pressure fluid system, and a pressure testing system. The fracture is set up by overlaying one high strength transparent polymethyl methacrylate slab over another. The size of the slab is $550 \times 65 \times 30 \mathrm{~mm}$. The area of the board is $650 \times 80 \mathrm{~mm}$ and the real seepage area $550 \times 65 \mathrm{~mm}$ as shown in Figure 9. In the experiment, the two boards were fixed on four sides, with water injecting holes and discharging holes at both ends of the upper plate in the longer direction. Ducts were made inside the injecting and discharging holes to guarantee the fluid injecting and discharging the intersection evenly. Pressure observation holes with 


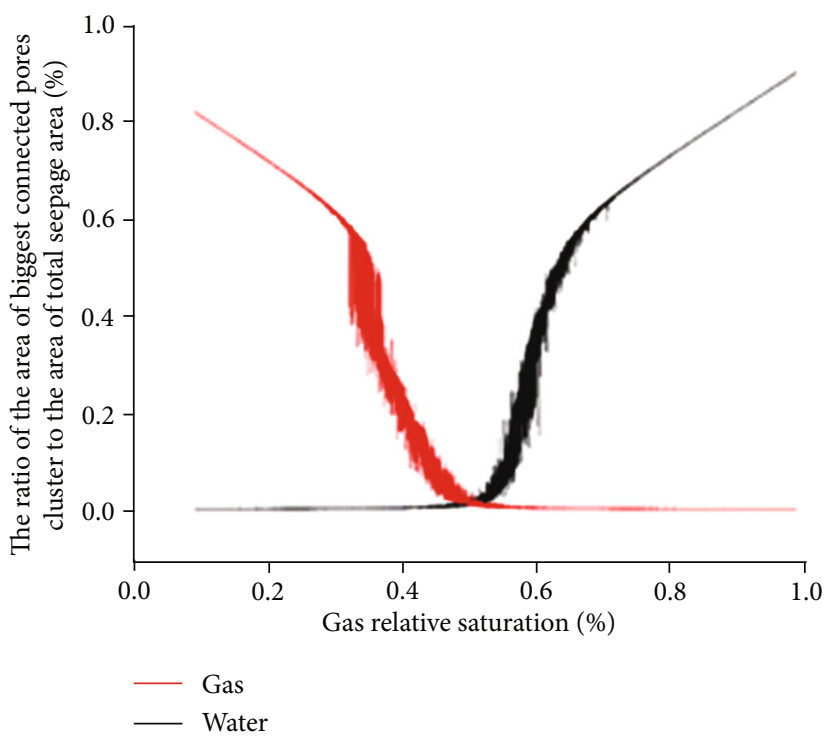

FIGURE 7: The proportion of gas-liquid's area of biggest cluster to total seepage area vs. gas relative saturation.

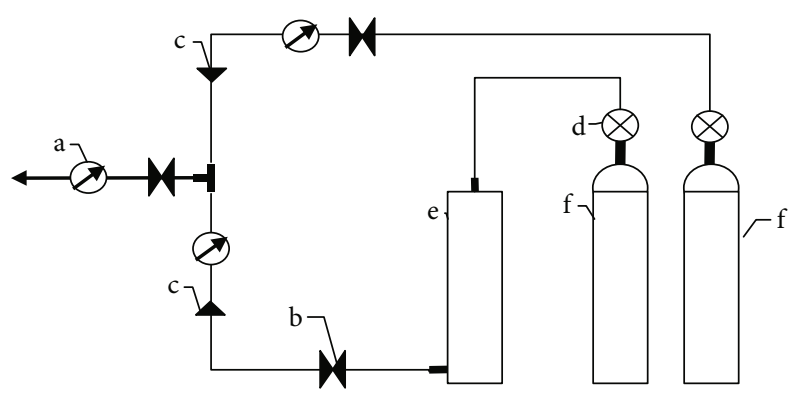

FIGURE 8: The sketch of pressure system (a) pressure gauge; (b) cutoff valve; (c) one-way valve; (d) pressure controller; (e) water cylinder; (f) compressed air cylinder.

$1 \mathrm{~mm}$ were drilled in the bottom plate along the flow direction. Standard fracture width and seal were made by using a feeler and seal belt. Pressure gauge, unidirectional valve, and cutoff valve were set in the injecting routes of gas and water. Pressure gauge and cut-off valve were set in the route of mixed fluid to control strictly the ratio between injected water and gas. Twenty-four pressure observation holes were drilled in scattered positions of the horizontal Hele-Shaw fracture experimental setup. The pressure of the gas-liquid fluid in fracture was tested by the high-resolution pressure gauge.

4.2. Experiment Procedure. When the pressure in the gas restore bottle and water bottle get to the given value, the cutoff valve of the mixing route was opened, and the gas-liquid two-phase fluid was injected into the fracture simultaneously. The gas discharge and water discharge in unit time were measured, and the relative saturation of gas and liquid was calculated. The saturation of gas and liquid was changed by adjusting the amount of water and gas injected to approach to the criterion or threshold value of the chaotic flow.
With the above method, numerous experiments of gasliquid two-phase flow with varied gas saturations in a plane fracture were carried out. The chaotic flow occurs when the gas saturation gets into a certain range. Then, the fluid flows stop, and the gas and water discharge cannot be tested. At this moment, a super-resolution digital camera was used to photograph the gas and liquid flow patterns in the fracture, and the area ratio of gas and liquid was analyzed to calculate the relative saturation of the gas and liquid.

4.3. Analysis of the Seepage Form of Gas-Liquid Flow in a Fracture. The experiment reveals that in fracture, the gaswater two-phase flow pattern is much more different from the flow pattern of liquid/gas one phase flow-uniform laminar flow. Figure 10 shows some flow patterns of gas and liquid observed in the experiment. When the two fluids flow in the same fracture, they constraint and promote each other, then complicated and sometimes chaotic flow would occur.

From Figure 10, we can see that the flow patterns in fracture are very complex due to the change of gas relative saturation. When the chaotic flow occurs, gas and water distribute disorderly in the fracture, which is far different from the flow state of one phase flow.

4.4. Measurement of the Permeability of the Gas-Liquid Flow. When the gas saturation approaches the critical value for the chaotic flow, the two-phase flow slows down and then stops. So, the saturation range for chaotic flow can be found by regulating the gas saturation to approaching to the chaotic flow. The experimental data were analyzed to compute the gas and liquid saturation and relative permeability. The gas emission and water discharge were measured at different gas saturation to calculate the relative permeability of gas or liquid. The experiment shows that the gas saturation is stable and regular out of the gas saturation range for chaotic flow. Figures 11 and 12 show the relation between the relative permeability and gas saturation when the fracture width is $0.24 \mathrm{~mm}, 0.1 \mathrm{~mm}$, and $0.05 \mathrm{~mm}$, respectively.

Figure 11 is the relation between gas saturation and permeability at the inlet pressure of $0.3 \mathrm{MPa}$ when the fracture width is $0.24 \mathrm{~mm}$. In these figures, the chaotic flow occurs when the relative gas saturation is from 42.9 to $56.8 \%$. The comprehensive seepage ability of the two-phase flow decreases to the lowest level at the gas saturation of $42.9 \%$ and then the gas saturation goes into the chaos range.

Figure 12 shows the relation between gas saturation and the relative permeability of two-phase fluid at the inlet pressure of $0.23 \mathrm{MPa}$ when the fracture width is $0.1 \mathrm{~mm}$. In this figure, the gas saturation for the chaotic flow is $48.8-62.3 \%$.

4.5. The Pressure Variation of the Two-Phase Flow. Figure 13 shows the pressure distribution corresponding photo of the gas-liquid flow patterns in the fracture when the chaotic flow takes place. The positive direction of the $x$-axis of the pressure distribution figure denotes seepage direction. The seepage directions of the two figures are all from left to right. The pressure is measured at different positions of the seepage field. 


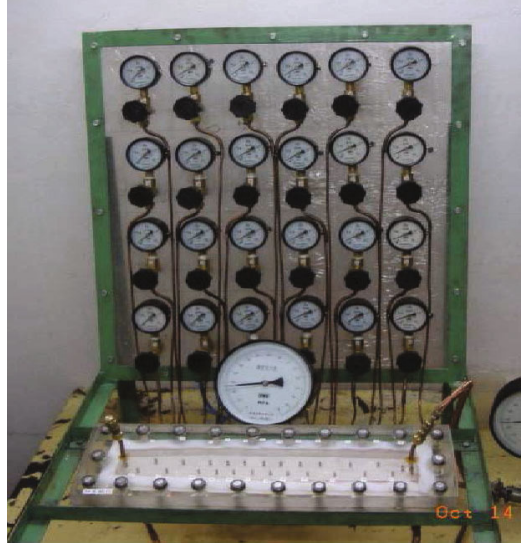

(a) Hele-Shaw simulating settings and pressure test device

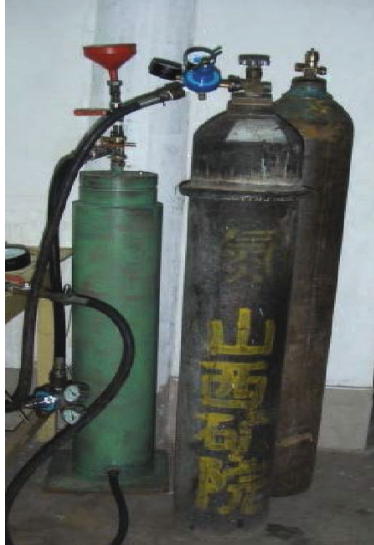

(b) Pressure gas cylinder and water cylinder

Figure 9: The simulating settings of gas-liquid two-phase flow in a fracture.

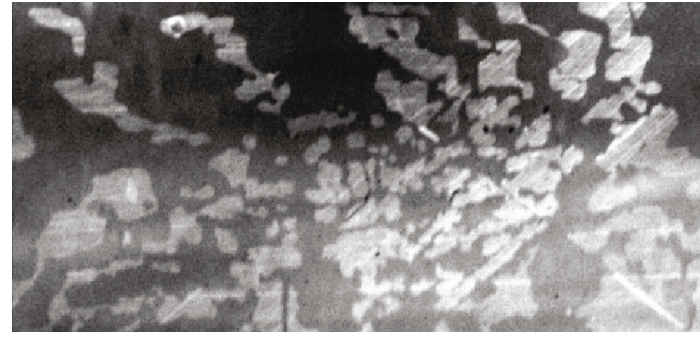

(a) Anomalistic gas clusters dispersed in water

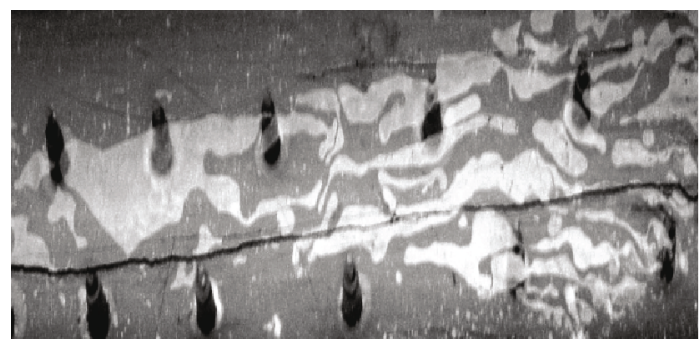

(c) Tortuous channel way of gas and water

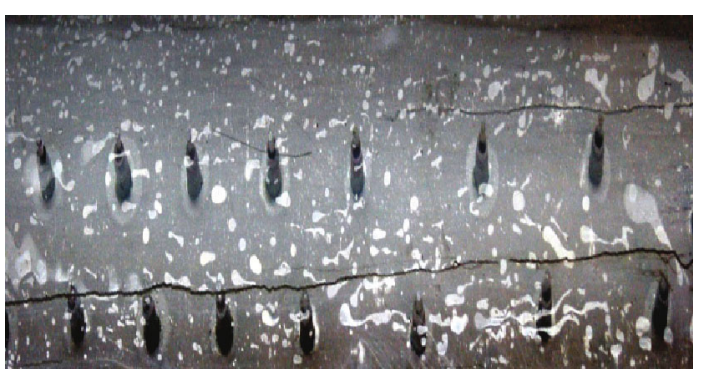

(b) Dispersed gas bubble

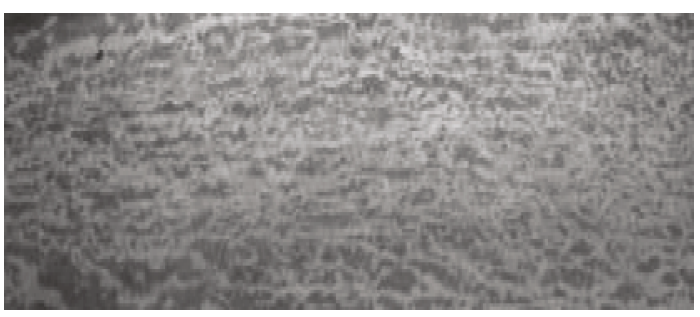

(d) The network route way of gas and water

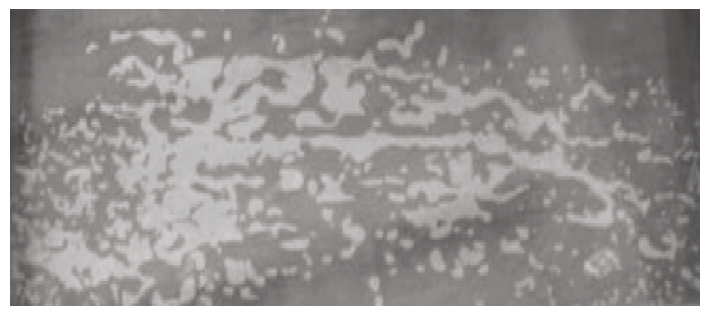

(e) The distribution of gas and water when critical seepage phenomena occur

Figure 10: Complex flow patterns of gas-liquid two-phase flow in fracture.

The inlet with much water (right side) in Figure 13(b) corresponds to the slowly decreasing pressure section in Figure 13(a). The interlaced gas and water in Figure 13(b) correspond to slightly varying pressure sections in Figure 13(a). By analyzing the areas of gas and liquid in Figure 13(b), the gas relative saturation is about $59.32 \%$.

In Figure 14, the gas is divided into three big clusters by water. The biggest gas cluster at inlet makes the fluid pressure decrease fast, which implied that the gas cluster absorbs a large amount of energy and the zero pressure gradients exist in a long section. By analyzing the areas of gas and water in the figure, the gas relative saturation is about $47.93 \%$.

By comparing the above two figures, it is found that in the gas-liquid chaotic flow in a real fracture, gas and liquid do not seepage independently but interlace in the finite fracture when their relative saturation gets to a threshold value. The 


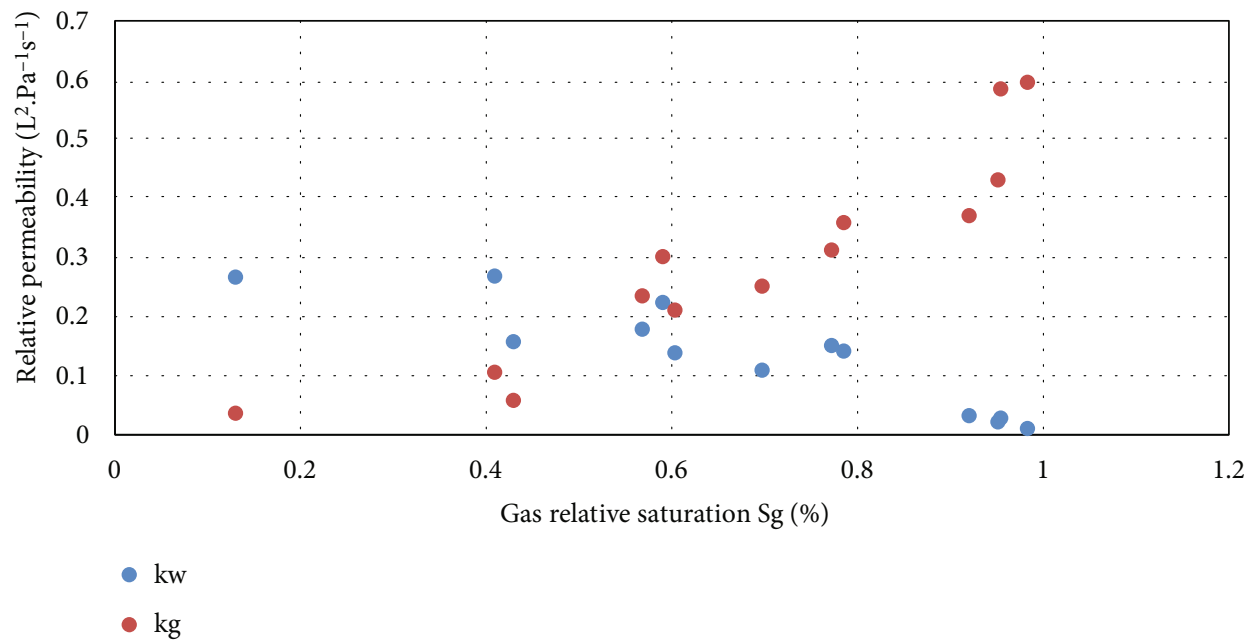

FIGURE 11: Gas-water relative permeability coefficient vs. relative gas saturation $\left(p_{\text {inlet }}=0.3 \mathrm{MPa}, b=0.24 \mathrm{~mm}, S_{\text {gcritical }}=42.9-56.8 \%\right)$.

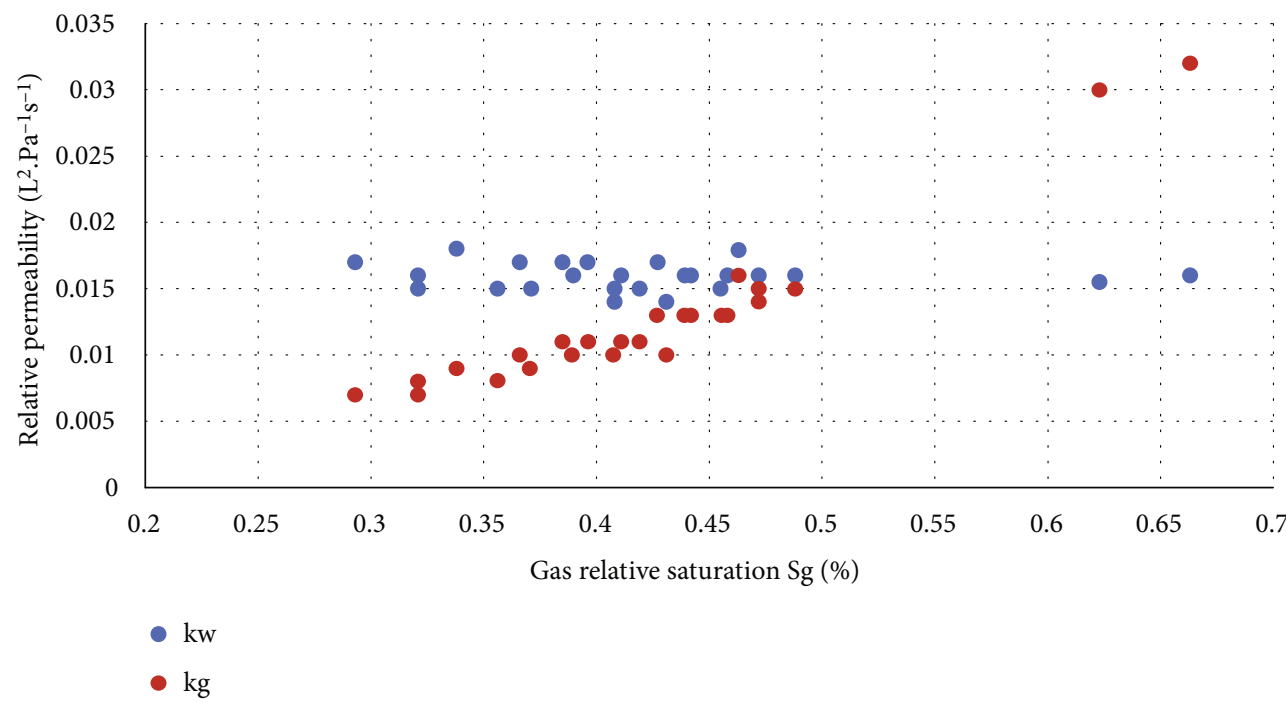

FIGURE 12: Gas-water relative permeability coefficient vs. relative gas saturation $\left(p_{\text {inlet }}=0.23 \mathrm{MPa}, b=0.1 \mathrm{~mm}, S_{\text {gcritical }}=48.8-62.3 \%\right.$ ).

repeated contracting and expending of the gas bubbles consumes a large amount of energy of the fluid. The pressure distribution is nearly horizontal, and the pressure gradient is zero. In this condition, the chaotic flow occurs.

4.6. The Threshold Range of the Chaotic Flow. The measured data in the above three experiments are listed in Table 1.

By analyzing the three groups of experimental data and other 200 photos of gas-liquid chaotic flow, it is found that the chaotic flow occurs when the relative gas saturation falls into the range from $43.9 \%$ to $63.47 \%$. It is supported by the experiment results that the gas relative saturation is the key factor affecting the two-phase flow patterns. Once the saturation of two-phase fluid gets into the saturation range for the chaotic flow, the gas-liquid flow will stop. It is measured in the experiment that the threshold gas saturation for the chaotic flow is from 43.9 to $63.47 \%$, which agrees well with the numerically simulated threshold saturation of $44 \%$ to $70 \%$.
The chaotic flow is a small probability event in the gas saturation of $64 \%-70 \%$, so it is hardly observed in the experiment and the experimental saturation range is smaller than the numerical simulation range.

\section{Conclusions}

In this paper, the numerical simulations and physical experiments were carried out to investigate the effect of the gas saturation on the gas-liquid chaotic flow. The mechanism of the chaotic flow is revealed, and the condition for its occurrence is identified. The main conclusions are as follows:

(1) The relative saturation of the gas-liquid fluid is a key factor controlling the gas-liquid seepage behavior. The gas-liquid flow form changes vastly with the relative saturation of gas. 


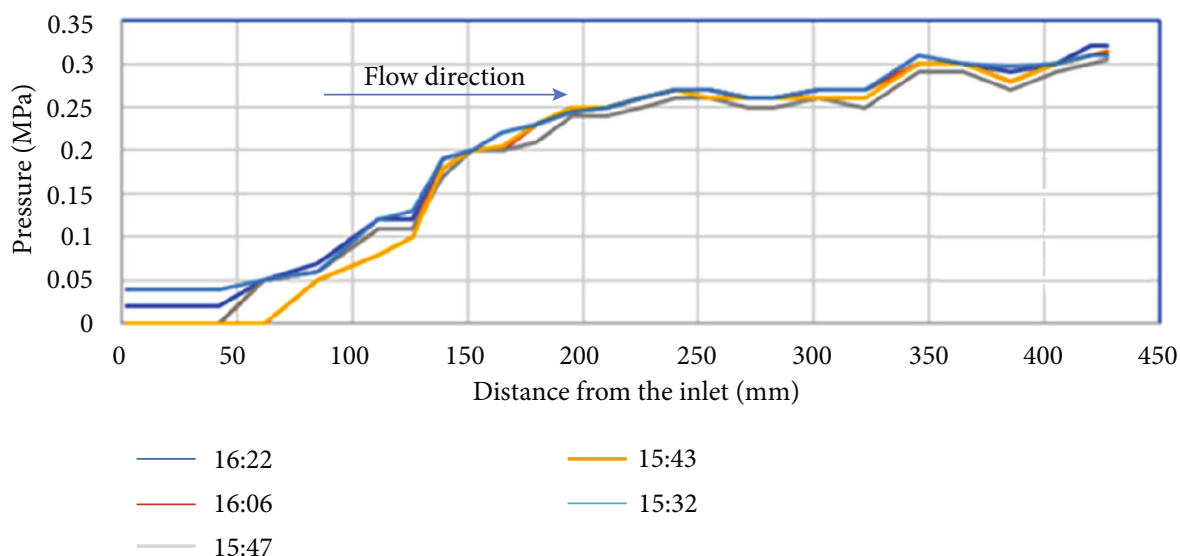

(a) Pressure distribution along the flow path

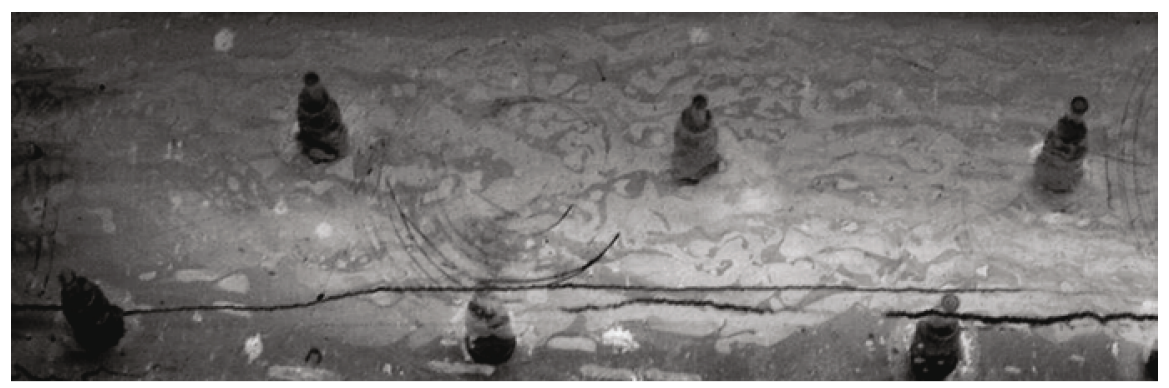

(b) Photograph corresponding to (a) when time is $15: 43$

FIGURE 13: Pressure distribution and its corresponding photograph.

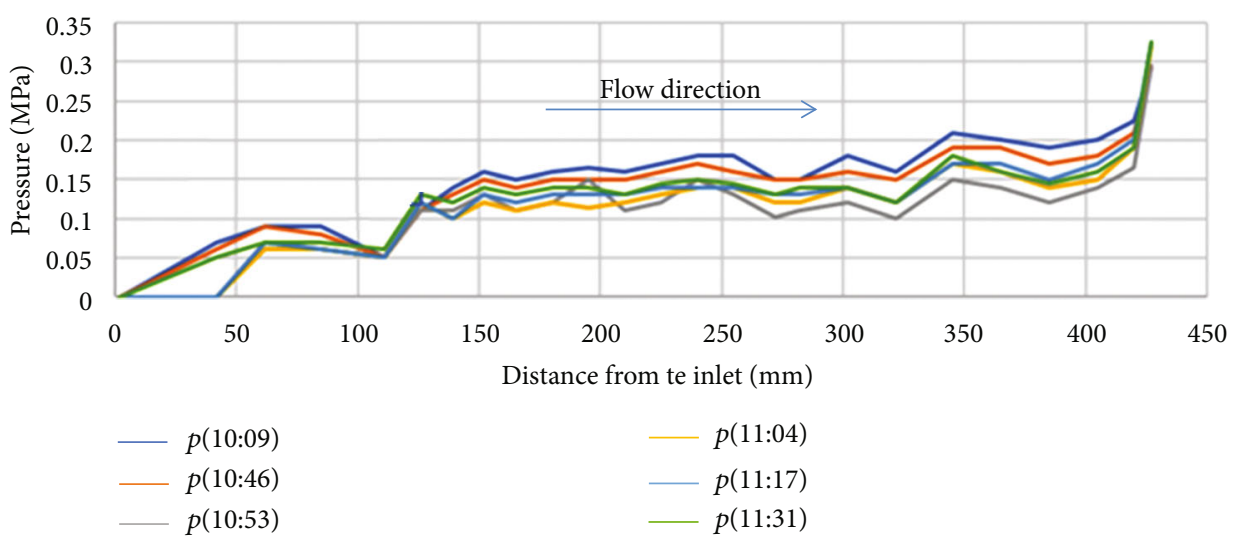

(a) Pressure distribution along the flow path

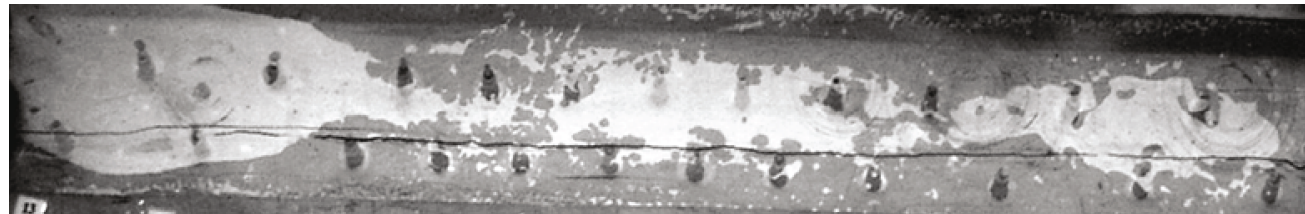

(b) Photograph according to (a) where time is $11: 04$

FIGURE 14: pressure distribution and its corresponding photograph.

(2) The gas-liquid chaotic flow may occur when the gas saturation reaches $44 \%-70 \%$, but the probability range larger than $80 \%$ occurs in the saturation ranging from $47 \%$ to $65 \%$. In the gas saturation of $57 \%-60 \%$, the probability of the chaotic flow is $100 \%$.
(3) The chaos of the gas-liquid flow happened from the enormous difference between the seepage properties of gas and liquid. Under the pressure, the gas cluster shrinks and expands repeatedly, which will restore and release energy repeatedly. The local nonstability and the complex interlace of gas and 
TABLE 1: Experimental data of gas-liquid chaotic flow in horizontal Hele-Shaw simulation.

\begin{tabular}{lcc}
\hline $\begin{array}{l}\text { Fracture width } \\
(\mathrm{mm})\end{array}$ & $\begin{array}{c}\text { Inlet pressure } \\
(\mathrm{MPa})\end{array}$ & $\begin{array}{c}\text { Gas saturation for chaotic } \\
\text { flow }\left(\mathrm{S}_{\mathrm{gc}} / \%\right)\end{array}$ \\
\hline 0.05 & 0.23 & $46.6-61.4$ \\
0.1 & 0.23 & $48.8-62.3$ \\
0.24 & 0.3 & $43.9-56.8$ \\
\hline
\end{tabular}

liquid occur in the seepage field. When the gas relative saturation gets to the threshold value, the gasliquid flow becomes disorder and shows a chaos form, the fluid pressure gradient becomes disorder, and the push force of the fluid is consumed up. At last, the gas-liquid two-phase flow turns into chaos and stops.

\section{Abbreviations}

$\rho_{\mathrm{w}}:$ Density of water $\left(\mathrm{g} / \mathrm{cm}^{3}\right)$

$\rho_{\mathrm{g}}:$ Density of gas $\left(\mathrm{g} / \mathrm{cm}^{3}\right)$

$q_{\mathrm{w}}:$ Specific velocity of water $(\mathrm{cm} / \mathrm{s})$

$q_{\mathrm{g}}$ : Specific velocity of gas $(\mathrm{cm} / \mathrm{s})$

$S_{\mathrm{w}}:$ Relative saturation of water (\%)

$S_{\mathrm{g}}: \quad$ Relative saturation of gas (\%)

$b$ : Fracture aperture $(\mathrm{cm})$

$s: \quad$ Local scale

$t$ : Time (s)

$\mu_{\mathrm{w}}:$ Dynamic viscosity of water (MPa s)

$\mu_{\mathrm{g}}:$ Dynamic viscosity of gas (MPa $\mathrm{s}$ )

$p: \quad$ Fluid absolute pressure (atm)

$p_{0}$ : Absolute pressure at outlet (atm)

$S_{\mathrm{g} 0}$ : Gas relative saturation when the fluid pressure is $p_{0}(\%)$

$M: \quad$ Mass of the gas $(\mathrm{kg})$

T: $\quad$ Temperature $(\mathrm{K})$

$R: \quad$ Gas constant, $R=8.3144598\left(\mathrm{~J} \cdot \mathrm{mol}^{-1} \cdot \mathrm{K}^{-1}\right)$

$Q_{\mathrm{w}}:$ Saturation of water $(\%)$

$Q_{\mathrm{g}}:$ Saturation of gas (\%).

\section{Data Availability}

All data used to support the study is included within the article.

\section{Conflicts of Interest}

The authors declare that there is no conflict of interest regarding the publication of this paper.

\section{Acknowledgments}

This work was supported by the National Key R\&D Program of China (Grant No. 2019YFA0705501, Grant No. 2019YFA0705502).

\section{References}

[1] R. F. Tangren, C. H. Dodge, and H. S. Seifert, "Compressibility effects in two-phase flow," Journal of Applied Physics, vol. 20, no. 7, pp. 637-645, 1949.

[2] P. G. Saffman and G. Taylor, "The penetration of a fluid into a porous medium or Hele-Shaw cell containing a more viscous liquid," Proceedings of the Royal Society of London. Series A. Mathematical and Physical Sciences, vol. 245, pp. 312-329, 1997.

[3] A. T. Corey, "The interrelation between gas and oil relative permeabilities," Producers Monthly, vol. 19, pp. 38-41, 1954.

[4] M. Ishii, Thermo-Fluid Dynamics Theory of Two-Phase Flow, Collection de la Direction Des études et Recherches d'Electricité de France 22, Eyrolles, Paris, France, 1975.

[5] S. I. Pai, Two-Phase Flows, Vieweg, Braunschweig, Germany, 1977.

[6] D. G. Avraam and A. C. Payatakes, "Generalized relative permeability coefficients during steady-state two-phase flow in porous media, and correlation with the flow mechanisms," Transport in Porous Media, vol. 20, no. 1-2, pp. 135-168, 1995.

[7] G. M. Homsy, "Viscous fingering in porous media," Annual Review of Fluid Mechanics, vol. 19, no. 1, pp. 271-311, 1987.

[8] E. Lajeunesse, J. Martin, N. Rakotomalala, and D. Salin, "3D instability of miscible displacements in a Hele-Shaw cell," Physical Review Letters, vol. 79, no. 26, pp. 5254-5257, 1997.

[9] P. Gondret, N. Rakotomalala, M. Rabaud, D. Salin, and P. Watzky, "Viscous parallel flows in finite aspect ratio HeleShaw cell: analytical and numerical results," Physics of Fluids, vol. 9, no. 6, pp. 1841-1843, 1997.

[10] K. A. Triplett, S. M. Ghiaasiaan, S. I. Abdel-Khalik, and D. L. Sadowski, "Gas-liquid two-phase flow in microchannels part I: two-phase flow patterns," International Journal of Multiphase Flow, vol. 25, no. 3, pp. 377-394, 1999.

[11] K. A. Triplett, S. M. Ghiaasiaan, S. I. Abdel-Khalik, A. LeMouel, and B. N. McCord, "Gas-liquid two-phase flow in microchannels," International Journal of Multiphase Flow, vol. 25, no. 3, pp. 395-410, 1999.

[12] N. P. Ekberg, S. M. Ghiaasiaan, S. I. Abdel-Khalik, M. Yoda, and S. M. Jeter, "Gas-liquid two-phase flow in narrow horizontal annuli," Nuclear Engineering and Design, vol. 192, no. 1, pp. 59-80, 1999.

[13] A. Kawahara, P. M.-Y. Chung, and M. Kawaji, "Investigation of two-phase flow pattern, void fraction and pressure drop in a microchannel," International Journal of Multiphase Flow, vol. 28, no. 9, pp. 1411-1435, 2002.

[14] D. T. Snow, "Anisotropie permeability of fractured media," Water Resources Research, vol. 5, no. 6, pp. 1273-1289, 1969.

[15] J. L. Xu, P. Cheng, and T. S. Zhao, "Gas-liquid two-phase flow regimes in rectangular channels with $\mathrm{mini} /$ micro gaps," International Journal of Multiphase Flow, vol. 25, no. 3, pp. 411432, 1999.

[16] M. K. Akbar, D. A. Plummer, and S. M. Ghiaasiaan, "On gasliquid two-phase flow regimes in microchannels," International Journal of Multiphase Flow, vol. 29, no. 5, pp. 855-865, 2003.

[17] A. Serizawa, Z. Feng, and Z. Kawara, "Two-phase flow in microchannels," Experimental Thermal and Fluid Science, vol. 26, no. 6-7, pp. 703-714, 2002.

[18] J. Yue, G. Chen, Q. Yuan, L. Luo, and Y. Gonthier, "Hydrodynamics and mass transfer characteristics in gas-liquid flow 
through a rectangular microchannel," Chemical Engineering Science, vol. 62, no. 7, pp. 2096-2108, 2007.

[19] E. Dana and F. Skoczylas, "Gas relative permeability and pore structure of sandstones," International Journal of Rock Mechanics and Mining Sciences, vol. 36, no. 5, pp. 613-625, 1999.

[20] P. G. Ranjith, S. K. Choi, and M. Fourar, "Characterization of two-phase flow in a single rock joint," International Journal of Rock Mechanics and Mining Sciences, vol. 43, no. 2, pp. 216223, 2006.

[21] S. Sisavath, X. D. Jing, and R. W. Zimmerman, "Effect of stress on the hydraulic conductivity of rock pores," Physics and Chemistry of the Earth, Part A: Solid Earth and Geodesy, vol. 25, no. 2, pp. 163-168, 2000.

[22] F. Skoczylas and J. P. Henry, "A study of the intrinsic permeability of granite to gas," International Journal of Rock Mechanics and Mining Sciences \& Geomechanics Abstracts, vol. 32, no. 2, pp. 171-179, 1995.

[23] Z. Wang, J. Feyen, and D. E. Elrick, "Prediction of fingering in porous media," Water Resources Research, vol. 34, no. 9, pp. 2183-2190, 1998.

[24] D. Yang, Y. Q. Hu, and Y. S. Zhao, "Theoretical and experimental studies on gas seepage in a single fracture under 3D stresses," Chinese Journal of Rock Mechanics and Engineering, vol. 23, pp. 999-1003, 2005.

[25] I. W. Yeo, M. H. De Freitas, and R. W. Zimmerman, "Effect of shear displacement on the aperture and permeability of a rock fracture," International Journal of Rock Mechanics and Mining Sciences, vol. 35, no. 8, pp. 1051-1070, 1998.

[26] M. González, M. Araujo, and A. Rodríguez, "Flow equations on a fractal structure," Physica A: Statistical Mechanics and its Applications, vol. 298, no. 3-4, pp. 297-314, 2001.

[27] B. Faybishenko, "Chaotic dynamics in flow through unsaturated fractured media," Advances in Water Resources, vol. 25, no. 7, pp. 793-816, 2002.

[28] E. M. Schlueter, R. W. Zimmerman, P. A. Witherspoon, and N. G. W. Cook, "The fractal dimension of pores in sedimentary rocks and its influence on permeability," Engineering Geology, vol. 48, no. 3-4, pp. 199-215, 1997.

[29] Y. L. Zhao, L. Y. Zhang, W. J. Wang, J. Z. Tang, H. Lin, and W. Wan, "Transient pulse test and morphological analysis of single rock fractures," International Journal of Rock Mechanics and Mining Sciences, vol. 91, pp. 139-154, 2017.

[30] Y. Zhao, Y. Wang, W. Wang, L. Tang, Q. Liu, and G. Cheng, "Modeling of rheological fracture behavior of rock cracks subjected to hydraulic pressure and far field stresses," Theoretical and Applied Fracture Mechanics, vol. 101, pp. 59-66, 2019.

[31] Y. Zhao, L. Zhang, W. Wang, W. Wan, and W. Ma, "Separation of elasto-visco-plastic strains of rock and a nonlinear creep model," International Journal of Geomechanics, vol. 18, no. 1, article 04017129, 2018.

[32] Y. L. Zhao, L. Y. Zhang, J. Liao, W. J. Wang, Q. Liu, and L. Tang, "Experimental study of fracture toughness and subcritical crack growth of three rocks under different environments," International Journal of Geomechanics, vol. 20, no. 8, article 04020128, 2020.

[33] Y. Zhao, L. Zhang, W. Wang, Q. Liu, L. Tang, and G. Cheng, "Experimental study on shear behavior and a revised shear strength model for infilled rock joints," International Journal of Geomechanics, vol. 20, no. 9, article 04020141, 2020. 